\title{
Governance na saúde: os desafios da operacionalização
}

\section{Governance in health: the implementation challenges}

\author{
Pedro Carrapato \\ (iD) https://orcid.org/0000-0002-2751-2247 \\ E-mail: pedro.carrapato®hbeatrizangelo.pt \\ Pedro Correiab \\ (iD) https://orcid.org/0000-0002-3111-9843 \\ E-mail: pcorreia®iscsp.ulisboa.pt

\section{Bruno Garciac} \\ (D) http://orcid.org/0000-0003-1502-7567 \\ E-mail: garcia.bruno.cœgmail.com \\ aHospital Beatriz Ângelo. Loures, Portugal. \\ bUniversidade de Lisboa. Instituto Superior de Ciências Sociais e \\ Políticas. Centro de Administração e Políticas Públicas. Lisboa, \\ Portugal. \\ 'Centro de Administração e Políticas Públicas. Lisboa, Portugal
}

\section{Resumo}

A massificação dos conceitos em geral torna-os, muitas vezes, difíceis de precisar. O conceito de governance tornou-se transversal a várias áreas, sendo orientado de acordo com a área em que é aplicado. Autores referem que a governance surge como um "chapéu" sob o qual se encaixam muitos temas, motivo pelo qual surgiram diversos conceitos, com influência das áreas em que eram aplicados. Embora pesem as diversas traduções para a língua portuguesa encontradas na literatura, de forma genérica, o termo "governance" pode ser entendido como um modelo de governação em rede. Este trabalho pretende percorrer as diversas definições de governance, governance associada ao setor da saúde e, dentro deste, os diversos conceitos de governance encontrados na literatura. O objetivo é perceber quais são os fatores que dificultam a operacionalização da governance na saúde. São descritos fatores que de forma persistente condicionam a operacionalização da governance. O desafio é encontrar formas inovadoras para conseguir atenuar o impacto desses fatores.

Palavras-chave: Governance; Saúde; Conceito; Operacionalização.

\section{Correspondência}




\section{Introdução}

The massification of concepts in general makes them often difficult to define. The concept of governance became popular across several areas, being orientated according to the area in which it is applied. Authors state that the governance emerges as a "hat" under which many themes fit, reason why several concepts were created, under the influence of the areas in which they were applied. In spite of the various translations into Portuguese in the literature, in a generic way, the term "governance" can be understood as a model of network governing. This work aims to approach the governance concept, governance associated with the health sector, and all the governance concepts associated to governance in health care. The aim is to understand the factors that stop the implementation of governance in health. There are factors hereinafter described that persistently affect the operationalization of governance. The challenge is to find innovative ways to mitigate the impact of these factors.

Keywords: Governance; Health; Concept; Implementation.
A imprecisão de conceitos é um dos problemas da análise científica. A utilização da palavra governance massificou-se, sendo algumas vezes aplicada de forma pouco criteriosa. A utilização de conceitos em geral, e do de governance em particular, requer precisão, de forma a adequá-lo ao contexto em que é utilizado. Uma das razões apontadas como justificação da popularidade do conceito é a sua ampla abrangência. Ao contrário de conceitos mais restritos, governance engloba uma vasta série de relações e instituições envolvidas no processo de governar (Raposo, 2008).

Apesar de o conceito de governance ser considerado tão antigo como a existência da governação, nos últimos 20 anos, ganhou destaque em áreas como as ciências sociais. As mudanças dos modelos de governance, no contexto português e europeu, levaram o Estado a deixar de exercer o poder de forma isolada. Surgem outros atores sociais de forma a dar resposta a problemas crescentes, como a falta de recursos, de legitimidade ou a crescente complexidade social (Rodrigues, 2013).

A utilização crescente do termo tornou-o transversal a diversas áreas, sendo orientado de acordo com o campo em que é aplicado. Tal como refere Bilhim (2013, p. 54), a governance aparece como "um guarda-chuva de coisas muito distintas".

Nesse sentido, o objetivo deste trabalho é percorrer as diversas definições de governance, governance associada ao setor da saúde e, dentro deste, os diversos conceitos de governance referidos na literatura. Pretende-se responder à seguinte questão: quais são os fatores que dificultam a operacionalização da governance na saúde? Tentarse-á descrever os mecanismos conhecidos para minimizar o impacto desses fatores na governance.

\section{Os conceitos de governance}

A multiplicidade de contextos e a "massificação" do termo exigem que se elaborem algumas considerações relativamente ao termo em si. As traduções do termo governance para o português têm o problema de não haver um termo equivalente para tradução direta; como tal, cada autor adota o 
termo que considera ser o mais correto. Por exemplo, no Relatório de Primavera (OPSS, 2003, p. 3) do Observatório Português dos Sistemas de Saúde, governance é traduzida por "governança" quando se refere aos "princípios que regem relações de poder capazes de promover os interesses efetivos do cidadão", e por "governação" quando é feita referência à "execução da ação do governo". Raposo (2008, p. 22) adota como tradução de governance o termo "governação", por entender que este evita "o problema depreciativo do termo governança e o neologismo governância”. Tradução idêntica é adotada por Bilhim (2004, p. 12) quando refere que “a governação (governance em língua inglesa) é um conceito que unifica a diversificada literatura sobre gestão pública e políticas públicas”. No sentido de evitar duplos sentidos na leitura deste trabalho, opta-se por não traduzir o termo e utilizar o termo inglês, governance.

A complexidade do conceito e a transversalidade de utilização do termo fizeram surgir múltiplas definições, influenciadas pelas áreas de estudo dos respetivos autores. Rhodes (1996) faz referência a pelo menos seis tipos de utilização do termo, ao mesmo tempo que relaciona governance a autoorganização, a redes interorganizacionais que se caracterizam pela interdependência enquanto estruturas do governo. No fundo, a governance é entendida como a governação em rede.

Sørensen (2002) também associa o conceito à governação em rede quando afirma que o exercício da governação se alterou consideravelmente nos últimos 30 anos. Gradualmente, assistiu-se a um "abandono" do modelo burocrático (sistemas hierarquicamente organizados que governam com recurso à lei) em prol de uma governança em rede, com modelos organizados de forma horizontal e relativamente fragmentados.

Outro autor que procurou encontrar um conceito de governance, Stoker (1998), entende que esta tem como objetivo criar condições para que existam regras ordenadas e ação coletiva. Os objetivos da governance não são diferentes do governo, afirmando o autor que a diferença está apenas no processo pelo qual se chega ao resultado. São identificados pelo autor cinco proposições que caracterizam o fenômeno da governance: a presença de instituições e atores dentro e fora do governo; a diluição das fronteiras e da responsabilidade na resolução de problemas sociais e econômicos; a identificação das relações de poder entre as instituições envolvidas na ação coletiva; a rede de atores com governação autônoma; e a capacidade de atingir os objetivos sem necessidade de recorrer ao poder ou ao uso da autoridade do governo. Stoker (1998) encara o governo como um utilizador de novas ferramentas ao seu dispor para dirigir e conduzir.

Três perspectivas distintas são identificadas por Pierre e Peters (200o apud Rodrigues, 2013): a analítica, na qual a governance é considerada enquanto quadro conceitual, e não como um fenômeno; a dinâmica, em que a governance é considerada um processo, atribuindo um papel privilegiado ao Estado enquanto coordenador e orientador; e uma terceira perspectiva, a estrutural, em que são privilegiados aspectos sociais da vida política e econômica, que compreende quatro conceitos de governance (hierarquias, mercados, comunidades e redes).

A governance como hierarquias engloba as estruturas estatais hierarquicamente verticalizadas (das quais o modelo burocrático é um exemplo). Quando a abordagem é do tipo mercado, o conceito de governance realça o caráter econômico e racional dos atores, e foca-se nos mecanismos por meio dos quais eles competem e cooperam. O modelo de governance como comunidades encontra-se associado a uma configuração governativa com uma participação mínima do Estado e um espírito cívico de participação reforçado. No último modelo referido, governance como redes, uma multiplicidade de atores participa e interage nos processos de concepção e implementação de políticas públicas (Rodrigues, 2013).

A análise de Kooiman (1999) sobre governance centra-se na relação entre a sociedade e o governo e sugere que a governance nas sociedades modernas se constitui por uma mescla das várias formas e/ ou modalidades utilizadas pelos vários atores para governar. $\mathrm{O}$ autor distingue três modos distintos de governance: autogovernance (sem ter um modelo imposto intencionalmente, caracteriza-se pela criação de redes de atores com regras e códigos próprios), cogovernance (estabelecimento vincado 
entre os conceitos de cooperação e colaboração, que podem fazer surgir cinco variedades de cogovernance) e governance hierárquica (o controle administrativo acontece associado ao cumprimento da lei, não só nos tribunais, mas também em formas sociais mais participativas). É entendido que a governance hierárquica não desapareceu totalmente das sociedades atuais, mas tem sofrido alterações e reinvenções (Kooiman, 1999; Rodrigues, 2013).

\section{Governance pela perspectiva das organizações internacionais}

Paralelamente aos múltiplos conceitos de governance, várias organizações internacionais introduziram o termo da governance no seu vocabulário. O World Bank (WB), por exemplo, introduz o tema aproximadamente em 1990, com a intenção de garantir uma utilização eficaz da assistência disponibilizada. Define governance como uma forma de poder que é exercido no processo de gestão dos recursos (sociais e econômicos) que influenciam o desenvolvimento do país (WB, 1993). Nanda (2006) cita Wolf, Bhagwati e Gambi (2005) para afirmar que a ajuda a países necessitados só será produtiva se tiver como destino países com boa governance, com uma responsabilidade política tolerável e uma boa performance.

Outro exemplo: a Organização das Nações Unidas (ONU), por meio do United Nations Development Programme (UNDP, 1997b), define governance como o exercício da autoridade política, econômica e administrativa quando se gerem os interesses de um país (Raposo, 2008).

Ambas as organizações internacionais mencionadas, para além do referido, definiram um conjunto de princípios para conseguir caracterizar a boa governance. Sucintamente, elas criaram dimensões para as quais tem que existir conformidade, de que são exemplo a luta contra a corrupção, a ausência de violência, a efetividade na governação, a preocupação com a qualidade da regulação, efetividade e eficiência, com a prestação de contas, estabilidade política, responsabilização, transparência e equidade (Ferreira; Raposo, 2006; Raposo, 2008).

\section{Corporate governance}

O conceito de corporate governance tem evoluído ao longo do tempo, muitas vezes como resposta a crises sistêmicas. O WB refere que um dos primeiros fracassos bem documentados de governance foi a South Sea Bubble (ou Companhia dos Mares do Sul) em 170o. De forma idêntica, grande parte da legislação relacionada com as leis de valores mobiliários nos Estados Unidos surge após a queda da bolsa de valores em 1929 (WB, 200o).

Historicamente, um número de falências de empresas conhecidas foi também associado à corporate governance. Cada episódio de crise ou falha foi associado a incompetência, fraude ou abuso na aplicação de novos elementos de um sistema de corporate governance. As empresas tornam-se instituições com poder e domínio, e conseguem alcançar o mundo com diferentes capacidades e formas de influenciar a economia. 0 crescente fenômeno da globalização conduz a maior perda de território, com a consequente diminuição de controle por parte do governo, o que se traduz em maior necessidade de prestação de contas (Abdullah; Valentine, 2009; WB, 2000).

Os autores Abdullah e Valentine (2009) referem que não é conhecida uma definição de corporate governance, mas que é possível associar o conceito a um conjunto de estruturas e processos utilizados para controlar e dirigir uma organização. No mesmo artigo, os autores concluem que a corporate governance pode ser entendida como um processo de relações sociais, em vez de um processo estruturado. O contexto empresarial atual implica, para além dos processos inerentes aos negócios, considerar outros fatores tidos como críticos, nomeadamente a legislação, a cultura e os contextos organizacionais.

$\mathrm{O}$ atual mundo, em constante desenvolvimento, acarreta desafios permanentes para a corporate governance. Tal implica que esta não seja explicada apenas por um corpo teórico, mas sim pela combinação de uma variação de teorias. Essa perspectiva permite uma abordagem não só das relações sociais, mas também de regras como a legislação e uma aplicação rigorosa das práticas de governance. Esse processo traduz-se numa abordagem que pretende "ir mais além” da aplicação mecânica de normas. A literatura 
tem descrito incumprimentos na corporate governance (apesar de existirem regras definidas), e o WB exemplifica-o com a Rússia (uma parte substancial dos lucros de uma empresa de óleos foi desviado pelo seu acionista, o que deixou a empresa em dívida com os seus credores, colaboradores e o Estado) e a Republica Tcheca (onde pequenos acionistas perderam todos os seus investimentos) (Abdullah; Valentine, 2009; WB, 2000).

\section{Governance na saúde}

De forma genérica, a governance em saúde consiste em promover e proteger a saúde da população a partir de ações e meios organizados com esse objetivo. A sua implementação passa por utilizar um sistema a que determinada sociedade recorre de forma a conseguir organizar e gerir os assuntos dos vários setores e parceiros, para que possa atingir os seus objetivos (Sakellarides, 2003; WHO, 1998a). A World Health Organization (WHO Organização Mundial da Saúde, em português) vai além ao fazer alusão à boa governance em saúde. Associa-a à transparência, à prestação de contas e a incentivos que promovam sistemas de saúde sustentáveis (WHO, 1998a).

A complexidade dos sistemas de saúde é inegável. Para além de lidarem com questões do interesse comum, "sofrem" da inevitabilidade dos problemas associados à influência dos determinantes da saúde, da "diluição" das fronteiras dos sistemas de saúde e dos próprios países. A liberdade atual de movimento das populações leva a que as políticas de saúde em nível nacional se mostrem insuficientes. Nesse sentido, tornou-se necessária a intervenção global como forma de responder às referidas necessidades emergentes no setor da saúde. Com o objetivo de estabelecer um novo paradigma na governance global no setor da saúde, em 1998 a WHO apoiou uma nova declaração, "Saúde para todos no século XXI" (Ferreira; Raposo, 2006; Raposo, 2008; Sakellarides, 2003; Zarpelon, 2012).

Tal como no conceito de governance, várias organizações internacionais incorporaram a governance na saúde ao seu vocabulário. O WB associa o conceito de governance ao conceito de saúde em vários dos seus relatórios. Em 1993, no relatório Investing in health (WB, 1993), é elaborada uma análise das relações entre saúde humana, política de saúde e desenvolvimento econômico. Por detrás dessa análise reside a certeza de que a "boa saúde" da população a torna mais produtiva, com repercussão no crescimento econômico do país. Investir na saúde é investir no desenvolvimento. 0 relatório aborda ainda questões controversas que envolvem cuidados de saúde, custos crescentes, iniquidade, ineficiência e políticas de saúde, associando-as ao desenvolvimento econômico. O WB sugere reformas na saúde baseadas em três tópicos, com o objetivo global de conseguir uma saúde para todos (WB, 1993).

Posteriormente, a obra Investing in health: development effectiveness in the health, nutrition, and population sector, de Johnston e Stout (1999), pretendeu avaliar o impacto dos programas de apoio desenvolvidos na área da saúde. Os autores assumem a necessidade de melhoria das políticas de saúde e de promoção de reformas no setor.

O programa referido anteriormente, UNDP, é utilizado pela ONU para relacionar a governance com o desenvolvimento humano, com destaque para as relações entre o Estado, a sociedade em geral e o setor privado. 0 documento Governance for sustainable human development: a UNDP policy document (UNDP, 1997a) destaca a importância do papel do Estado em áreas como a manutenção das condições de saúde pública, a manutenção dos padrões de segurança, e a criação de condições para garantir os direitos dos mais vulneráveis para toda a população. O documento faz referência a empowerment, cooperação, equidade, sustentabilidade e segurança como fatores fundamentais para o desenvolvimento humano. Identifica ainda a visão estratégica, a efetividade, a eficiência, a prestação de contas, a transparência e a equidade como características principais da boa governance.

A WHO dirige o seu entendimento de governance em sentido idêntico ao da ONU. O desenvolvimento, o desempenho dos sistemas de saúde, a transparência e a prestação de contas são vetores foco da organização quando se aborda a questão da governance. Associado ao exposto, é uma organização que explicitamente aborda a questão da governance em saúde e da boa governance em saúde. 
Pode se considerar os seguintes documentos publicados pela WHO como um espelho do seu entendimento de governance em saúde.

No documento Health 21: health for all in the 21st century, a WHO (1998b) assume que conseguir um potencial de saúde para todos é uma meta constante. São definidos dois objetivos principais: a promoção e proteção da saúde das pessoas ao longo de suas vidas; e a redução da incidência das principais doenças e lesões para aliviar o sofrimento que causam. A fundamentação ética do documento consiste em três valores considerados básicos: a saúde como um direito humano fundamental; a equidade em saúde e a solidariedade na ação entre e dentro de todos os países, como também das respectivas populações; e a participação e responsabilização de indivíduos, grupos, instituições e comunidades para o desenvolvimento da saúde continuada.

Nesse documento a WHO (1998b) define ainda 21 metas de saúde que espelham as necessidades de toda a região europeia, com sugestões de medidas consideradas necessárias para melhorar sua situação. Estas vão funcionar também como instrumento de benchmarking, com o objetivo de medir a evolução na melhoria e proteção da saúde e na redução dos riscos de saúde. As referidas metas, em conjunto, funcionariam como estrutura base para desenvolver políticas de saúde nos países da região europeia.

A publicação Good governance for health (WHO, 1998a) vem abordar questões relacionadas com a crise nos sistemas de saúde e as consequências da má governance no setor. A incapacidade de recursos humanos, técnicos e financeiros é indicada como causa para a crise dos sistemas de saúde, provocando dificuldade no acesso aos cuidados de saúde e diminuição na satisfação por parte da população com os serviços e prestadores de cuidados. Isso diminui a capacidade de responder às necessidades crescentes do setor (WHO, 1998a).

0 mesmo documento refere que a resposta tendencial à crise nos sistemas de saúde tem sido tratar os sintomas dos problemas, em vez de "apontar" para a sua fonte. Tratar os sintomas dos problemas resulta em soluções temporárias e por vezes dispendiosas. 0 que está por detrás da referida crise dos sistemas de saúde são questões muito mais complexas do que podem parecer quando o foco são os sintomas isolados. A WHO sugere no documento que, para se conseguir ultrapassar a natureza do problema, será necessário analisar as práticas de governance. É indicado no relatório que colocar quem necessita dos cuidados de saúde no centro do sistema de saúde, em vez dos fornecedores de cuidados, é o caminho para uma boa governance (WHO, 1998a).

No ano 200o, a WHO publicou o relatório Health systems: improving performance, o qual, para além de abordar temas relacionados com a governance, enfoca o desempenho dos sistemas de saúde. Tem como propósito melhorar e proteger a saúde, não descurando alguns aspectos intrínsecos, como a justiça no pagamento dos cuidados de saúde e as expectativas das pessoas no que diz respeito à forma como são tratadas. Garantir um nível médio de cuidados de saúde é insuficiente, pois um sistema de saúde deve estar focado também na redução das desigualdades, na tentativa de melhorar a situação dos mais desfavorecidos. Nesse documento é introduzido o conceito de stewardship, que pretende reforçar o papel e a responsabilidade do governo na governance da saúde (o conceito de stewardship será explorado mais à frente).

Em 2002, a WHO publicou The European health report 2002, em que é elaborada uma análise geral da situação da saúde na Europa, com foco na esperança média de vida, na mortalidade e em doenças de comunicação obrigatória. 0 documento aborda os fatores socioeconômicos, ambientais e de estilos de vida enquanto principais determinantes da saúde, sem deixar de fora da análise as reformas e políticas dos sistemas de saúde. Refere existirem transformações dos sistemas de saúde da região europeia, ainda que continuem a existir questões por resolver relacionadas com a equidade de alguns grupos da sociedade. Apesar de se ter verificado um esforço para o equilíbrio da relação entre a sustentabilidade e o financiamento, os problemas com a primeira traduzem-se em diminuições da acessibilidade e da disponibilidade dos serviços de saúde. O conceito de stewardship é novamente focado e é reforçada sua importância.

O relatório de 2006, Working together for health (WHO, 2006), dirige-se ao problema transversal da escassez de recursos humanos (tema já abordado em 
documentos anteriores). Para além dos países em desenvolvimento, a falta crônica de profissionais de saúde bem treinados existe também nos países desenvolvidos, tornando o tema um problema global. Um conjunto de razões relacionadas com migração, doença ou morte tornou os países incapazes de "produzir" recursos especializados na área da saúde. O documento aponta que a solução para esse problema não reúne consenso, mas que a sua correção passa pela cooperação entre as partes interessadas e envolvidas no processo (WHO, 2006).

Em 2010, a WHO publicou o relatório Health systems financing: the path to universal coverage, dedicado à cobertura universal dos cuidados de saúde. Apesar de esse ser um objetivo partilhado por muitos países, existem diferenças na interpretação do conceito em si. Segundo a WHO, ter um sistema de cuidados de saúde com total cobertura implica a existência de um sistema de financiamento sustentável. Isso significa garantir que os sistemas de financiamento suportem as necessidades de cuidados de saúde da população para que o acesso aos cuidados não coloque o utilizador numa situação de carência financeira. São definidos no relatório três eixos de cobertura: a população (cobertura dos seus serviços e proteção financeira); os serviços; e os custos (fundamental para a proteção financeira), com a garantia de cuidados de saúde de qualidade.

O documento Health 2020: a European policy framework and strategy for the 21st century, publicado em 2013 pela WHO, pretende criar uma estrutura política comum entre os Estados-membros da organização. Tem como objetivo melhorar o bemestar das populações, diminuir as desigualdades no setor da saúde, fortalecer a saúde pública e garantir um sistema de saúde centrado nas necessidades da população, universal, sustentável, com equidade e qualidade. 0 documento reforça a importância da cooperação além-fronteiras como forma de resposta aos novos desafios no setor, e ao mesmo tempo mantém a preocupação com a equidade, não só no tempo atual, mas também nas gerações seguintes. É notória a preocupação com a manutenção das estratégias enunciadas nas gerações futuras e o reforço de que os governos devem estar envolvidos/ comprometidos ativamente em iniciar abordagens para a melhoria da saúde e de sua qualidade.

\section{A associação do conceito de governance dentro do setor da saúde}

A governance na saúde é um foco cada vez maior em nível nacional e internacional. Pela necessidade de desenvolvimento dos sistemas de saúde e de implementação de reformas, tem-se adotado o conceito de governance quer orientado para questões relativas à prática clínica, quer para questões relacionadas com os órgãos de governo das organizações.

\section{Governance clínica}

A governance clínica é entendida por Gonçalves (2005) como um processo bottom-up, desenvolvido localmente, com o objetivo de motivar os profissionais de saúde a construir parcerias efetivas com os doentes. Originária do National Health Service (NHS) do Reino Unido, pela melhoria dos padrões da prática clínica por meio de um conjunto de processos (prática clínica baseada em evidência científica, monitorização clínica, desenvolvimento e pesquisa, auditoria, gestão do risco, envolvimento dos doentes, eficácia clínica) (Starey, 2001). Autores céticos quanto a essa perspectiva reclamam que os objetivos da governance clínica não trazem nada de inovador. Os argumentos apontam que a ela não é mais do que a confirmação daquilo que se espera dos profissionais de saúde (Goodman, 1998).

\section{Governance integrada}

Um conceito também associado ao NHS, busca enquadrar diferentes tipologias de organizações de forma a conseguirem atingir os objetivos organizacionais, de segurança e qualidade dos serviços prestados. Nessa definição prevê-se a prestação de contas aos doentes, à comunidade em geral e aos parceiros organizacionais (Deighan; Bullivant, 2006). Raposo (2008) afirma que essa abordagem tem como objetivo unir diferentes áreas da governance, atribuindo aos órgãos de administração um papel central, devendo ter presente, em suas decisões, o envolvimento dos cidadãos. A tomada de decisão deverá ter em conta o impacto que causará na comunidade que recorre à organização de saúde. 


\section{Governance hospitalar}

A complexidade organizacional associada aos hospitais é inegável. No setor da saúde, é a organização que utiliza de forma mais intensa o capital humano e tecnológico, e o conhecimento no cumprimento do seu papel - a prestação de cuidados de saúde.

A literatura aborda vários fatores quando a questão é governance hospitalar. Shortell (1989), no seu artigo "New directions in hospital governance", faz referência a três aspectos que devem ser considerados para se evoluir em direção à governance hospitalar. Os conselhos de administração (CA) necessitam de elementos peritos em áreas como marketing, legislação e finanças. A presença de peritos da área clínica também é descrita como fundamental por serem estes os responsáveis pela prestação de cuidados de saúde. 0 segundo aspecto referido está relacionado com a necessidade de simplificar a tomada de decisão, algo conseguido com menos membros nos CA, mas mais ativos e focados. A existência de grupos de planejamento estratégico e de qualidade é mandatória. A necessidade de os hospitais diminuírem sua preocupação com os problemas dos processos e passarem a estar orientados para os resultados, mesmo que isso implique correr riscos, é considerada pelo autor como o terceiro aspecto (Shortell, 1989).

A maioria dos hospitais necessita de uma estrutura administrativa eficiente e de um indispensável CA. Em conjunto, estes são entendidos como a coluna vertebral do processo de governance hospitalar. Este consiste no processo de governar o funcionamento global do hospital, de acordo com sua missão e objetivos, simultaneamente monitorizando o nível de realização operacional. A essência da governance hospitalar é entendida como a gestão dos processos e o apoio na prestação de cuidados, com o objetivo de ganhos em saúde, não descurando os critérios de avaliação (Eeckloo et al., 2004). Essa definição permite associar a governance hospitalar à supervisão de todas as atividades dos hospitais, incluindo o desempenho clínico, contido na governance clínica abordada anteriormente.
A pesquisa empírica sobre governance hospitalar realizada por Eeckloo et al. (2004) concluiu que o número limitado de pesquisas existentes na literatura era de origem anglo-saxônica. Nessas pesquisas encontrou como tópicos de governance hospitalar: a relação entre a estratégia do hospital e a composição do CA; as diferenças entre os vencimentos dos membros do CA em instituições sem e com fins lucrativos; a influência do desempenho global do hospital e o turnover dos membros do CA; e por último, que as diferentes formas organizacionais têm um grande impacto na governance do hospital. Esse último tópico é difícil de generalizar para um contexto diferente do sistema de saúde americano pelas suas características específicas.

A mesma pesquisa clarificou que nos hospitais os objetivos são muitas vezes contraditórios. Por um lado o objetivo principal do hospital é fornecer cuidados de saúde com qualidade, mas não pode descurar a acessibilidade aos cuidados de saúde e ao equilíbrio financeiro do próprio hospital (Eeckloo et al., 2004).

As alterações na sociedade moderna, fruto do desenvolvimento econômico, da globalização, do desenvolvimento científico e tecnológico, do aumento do acesso à informação e do reconhecimento dos direitos dos consumidores, levaram à percepção de que o comportamento ético é fundamental no desempenho das organizações. As questões referidas encontram-se sob a "alçada" do conceito de responsabilidade social. Recentemente esse conceito tem sido associado ao setor da saúde, mais concretamente ao contexto da prestação de cuidados de saúde, sugerindo um novo paradigma na gestão hospitalar (Brandão et al., 2013).

\section{Stewardship}

$\mathrm{Na}$ literatura surge um conceito associado ao de governance na saúde, stewardship, introduzido com o objetivo de reforçar o papel do Estado, do governo e, consequentemente, do ministro da saúde na governance desse setor (WHO, 2000, 2002). 0 conceito tem por base a stewardship theory que, fundamentada em perspectivas sociopsicológicas, estuda as relações em organizações em que os 
colaboradores valorizam mais comportamentos cooperativos do que comportamentos motivados pelo interesse próprio. Quando os indivíduos estão alinhados com os interesses da organização, a sua produtividade terá mais utilidade para a organização do que quando trabalham com objetivos próprios. Essa teoria tem por base um modelo sustentado na necessidade de eficiência e efetividade, em que o agente pretende essencialmente fazer um bom trabalho, alinhado com os objetivos do principal (Schillemans, 2013; Welsh et al., 2013).

O conceito introduzido pela WHO pretende reforçar o papel do principal (Estado, governo, ministro da saúde) na governance na saúde enquanto coordenador e orientador de ações a serem adotadas pelos agentes (sistema de saúde, prestadores de cuidados de saúde). Esse conceito tem implícita, para além da capacidade que o principal tem de influenciar o agente na concretização dos objetivos dos sistemas de saúde, uma dimensão ética relacionada com a responsabilização pelo bem comum e uma relação de confiança com o cidadão. A stewardship pressupõe a formulação de políticas de saúde que se traduzem na visão e na direção que o sistema de saúde adota (Raposo, 2008).

\section{Smart governance}

No atual mundo do conhecimento e da tecnologia, as decisões puramente normativas perdem terreno para decisões baseadas nas evidências. Paralelamente, as tomadas de decisão exigem novos métodos para conseguir lidar com o acúmulo de incertezas resultantes da evolução do conhecimento.

0 termo smart governance surge em 2007 a partir de uma obra de Willke, que a define como o grupo de ações conjuntas que constituem um modelo de governance com capacidade de resposta para as exigências de uma sociedade do conhecimento. Os atuais governos, de forma a manter a sua legitimidade e conseguir melhorar o seu desempenho, necessitam de novas formas de governance, que contemplem atores dentro e fora do governo. A smart governance em saúde entende formas mais amplas de compreensão da saúde e pressupõe mudanças na relação conjunta de trabalho entre o Estado e a sociedade (Kickbusch; Gleicher, 2012).

A smart governance vem definir a forma estratégica como os governos podem abordar os novos desafios no setor da saúde. São apontadas cinco dimensões para fazê-lo: a colaboração; o envolvimento; uma combinação entre regulação e persuasão; e o recurso a agências independentes e organismos especializados, com políticas adaptativas, estruturas resilientes e previdência. A smart governance é entendida como a expansão da boa governance, com a inclusão de dimensões como a saúde e o bem-estar. Valores como direitos humanos, bem-estar, justiça e equidade social tornam-se os princípios éticos que orientam a elaboração das políticas de saúde (Kickbusch; Gleicher, 2014).

\section{Desafios da governance na saúde}

Atualmente é percetível por parte dos governantes que os recursos destinados ao setor da saúde não alcançam os resultados pretendidos. Para que isso aconteça é necessário dar prioridade à governance. Danila e Mohamed (2013) referem que, na ausência dessa prioridade, se correrá o risco de não se utilizarem os fundos destinados ao setor de forma eficaz, com a consequente inviabilização dos objetivos delineados.

O reconhecimento internacional da necessidade de uma entrega de cuidados de saúde bemsucedida, dependente de instituições com uma gestão efetiva, conduziu a uma ênfase da governance na saúde por parte de governantes, da academia e das organizações internacionais. Estão descritos na literatura princípios que podem ser utilizados no sentido da operacionalização da governance; no entanto, a sua compreensão é importante e necessária no desafiante contexto dos sistemas de saúde atuais (Barbazza; Tello, 2014).

No Quadro 1 encontram-se os princípios de governance e a respectiva forma de os operacionalizar. 


\section{Quadro I - Princípios de governance dos sistemas de saúde}

\begin{tabular}{|c|c|}
\hline Principio de governance & Operacionalização \\
\hline Visão estratégica & $\begin{array}{l}\text { Os líderes devem ter uma visão clara dos objetivos pretendidos em curto e longo prazo sobre a } \\
\text { saúde e o desenvolvimento humano. Paralelamente devem existir planos de ação para realizar } \\
\text { esses objetivos. }\end{array}$ \\
\hline Participação e consenso & $\begin{array}{l}\text { Todos os homens e mulheres devem ter a possibilidade de determinar a sua escolha para a } \\
\text { saúde, de forma direta ou indireta (através de instituições intermediárias que representem seus } \\
\text { interesses). A boa governance dos sistemas de saúde permite que perspectivas divergentes sejam } \\
\text { discutidas de forma a alcançar um consenso (por meio de reuniões abertas, workshops públicos, } \\
\text { inquéritos de satisfação). }\end{array}$ \\
\hline Cumprimento da lei & $\begin{array}{l}\text { As estruturas legais relativas à saúde (legislação, regras, procedimentos, códigos de conduta, } \\
\text { acreditação) devem ser justas e aplicadas de forma imparcial. }\end{array}$ \\
\hline Transparência & $\begin{array}{l}\text { Fluxo livre de informações para todos os assuntos relacionados com a saúde (liberação de informações } \\
\text { de desempenho, serviços de inspeção, disponibilização de informação orçamental e financeira). }\end{array}$ \\
\hline Receptividade & $\begin{array}{l}\text { Instituições e processos devem servir a todas as partes interessadas, de forma a garantir que as } \\
\text { políticas e os programas respondam às necessidades de saúde em geral, e não às necessidades de } \\
\text { saúde específicas dos seus clientes. }\end{array}$ \\
\hline Equidade e inclusão & $\begin{array}{l}\text { Todos os indivíduos, sem exceção, devem ter oportunidades para melhorar ou manter sua saúde } \\
\text { e bem-estar. }\end{array}$ \\
\hline Eficácia e eficiência & $\begin{array}{l}\text { Processos e instituições devem produzir resultados que respondam às necessidades da } \\
\text { população. Os resultados de saúde devem ter impacto na otimização dos recursos. }\end{array}$ \\
\hline Prestação de contas & $\begin{array}{l}\text { Prestação de contas do ministério da saúde e de outras agências governamentais relevantes (sistemas } \\
\text { de combate à corrupção, contratos baseados em resultados, pagamento por desempenho). }\end{array}$ \\
\hline Inteligência e informação & $\begin{array}{l}\text { Inteligência e informações são cruciais para a compreensão do sistema de saúde. Devem ser } \\
\text { tomadas decisões informadas, que respondam às necessidades de diferentes grupos de interesse } \\
\text { (relatórios elaborados por pesquisadores independentes, auditorias periódicas, avaliações de } \\
\text { desempenho, utilização de ferramentas reconhecidas internacionalmente para análise de situação). }\end{array}$ \\
\hline Ética & $\begin{array}{l}\text { Questões referentes à ética em cuidados de saúde incluem o respeito pela autonomia, a não } \\
\text { maleficência, a beneficência e a justiça. }\end{array}$ \\
\hline
\end{tabular}

Fonte: Barbazza e Tello (2014, p. 7); Danila e Mohamed (2013, p. 6)

Não obstante a existência de princípios definidos e de formas de operacionalização da governance, seus objetivos continuam não alcançados. 0 difícil progresso da saúde global é atribuído por alguns autores (Barbazza; Tello, 2014; Frenk; Moon, 2013; Gostin; Mok, 2009) a questões consideradas desafiantes. Um dos exemplos (fruto da globalização) consiste na atual dificuldade em controlar a livre circulação de pessoas, com a consequente incapacidade de deter o impacto que os determinantes da saúde podem ter na saúde (veja-se, por exemplo, Carrapato; Correia; Garcia, 2017). É descrita uma tensão entre a realidade de cada país e as orientações/recomendações/ imposições de ação coletiva internacional. A diferente distribuição de recursos de saúde, diferentes interesses dos diferentes intervenientes, a diversidade cultural e a rápida mudança de poder entre países tornam ainda mais complexa a operacionalização da governance (Frenk; Moon, 2013; Gostin; Mok, 2009).

Na ausência de um governo global, é necessário que cada país concorde com as suas responsabilidades, obrigações, direitos e deveres. Como consequência dessa responsabilização surgirão respostas mais eficazes às ameaças da saúde comum (Frenk; Moon, 2013). São identificadas por Gostin e Mok (2009, p. 9) seis áreas consideradas vitais para a saúde mundial (com necessidade de intervenção), apontadas no Quadro 2. 


\section{Quadro 2 - Áreas com necessidade de intervenção}

\begin{tabular}{|c|c|}
\hline Área com necessidade de intervenção & Observações \\
\hline A falta de liderança em saúde global & $\begin{array}{l}\text { A WHO é a organização com reconhecimento para uma liderança global na } \\
\text { saúde. Apesar do importante papel por ela desempenhado, algumas críticas } \\
\text { são "lançadas" quanto à sua capacidade de liderança na promoção da saúde } \\
\text { em nível internacional (exemplo dado pela ausência de emissão de tratados até } \\
2003 \text {, quando da publicação da Framework Convention on Tobacco Control). }\end{array}$ \\
\hline $\begin{array}{l}\text { A necessidade de aproveitar a } \\
\text { criatividade, energia e recursos para a } \\
\text { saúde global }\end{array}$ & $\begin{array}{l}\text { A multiplicidade de atores envolvidos no objetivo da saúde global pode ser um } \\
\text { fator com impacto positivo, desde que esses atores se mantenham empenhados } \\
\text { e coordenados na melhoria da saúde global (exemplo dado com potenciais } \\
\text { benefícios que podem advir de empresas privadas, com a inovação de produtos } \\
\text { farmacêuticos. Podem ainda ser concebidos meios de criação de incentivos, de } \\
\text { forma a facilitar, coordenar e canalizar atividades desses atores não estatais). }\end{array}$ \\
\hline $\begin{array}{l}\text { A falta de colaboração e coordenação } \\
\text { entre vários atores }\end{array}$ & $\begin{array}{l}\text { A colaboração e coordenação entre os vários intervenientes na saúde global é um } \\
\text { problema considerado. } 0 \text { aumento de atores envolvidos resulta em problemas } \\
\text { de fragmentação e duplicação nos financiamentos, programas e atividades no } \\
\text { domínio da saúde global. }\end{array}$ \\
\hline $\begin{array}{l}\text { A negligência de necessidades básicas } \\
\text { de sobrevivência e fortalecimento dos } \\
\text { sistemas de saúde }\end{array}$ & $\begin{array}{l}\text { o desenvolvimento dos sistemas de saúde sustentáveis de forma a suprimir as } \\
\text { necessidades básicas tem sido negligenciado, traduzindo-se num problema na } \\
\text { saúde global. Essas necessidades são essenciais para recuperar a capacidade } \\
\text { humana (são exemplos o saneamento, o controle de pragas, ar e água puros, e o } \\
\text { controle do tabagismo, dieta e nutrição). }\end{array}$ \\
\hline $\begin{array}{l}\text { A questão da definição de financiamento } \\
\text { e prioridade }\end{array}$ & $\begin{array}{l}\text { O problema de prioridades distorcidas no financiamento internacional é outro } \\
\text { desafio. Atualmente, parte do financiamento é dirigido para doenças específicas } \\
\text { ou interesses de segurança nacional (por exemplo, quase } 70 \% \text { do orçamento } \\
\text { da ajuda global dos EUA para a saúde é dedicado ao HIV). É necessária uma } \\
\text { abordagem de cooperação mais forte entre os países que doam e os países } \\
\text { beneficiários. }\end{array}$ \\
\hline $\begin{array}{l}\text { A necessidade de prestação de contas, } \\
\text { transparência, monitorização e } \\
\text { fiscalização }\end{array}$ & $\begin{array}{l}\text { Existe uma necessidade, considerada crítica, de maior transparência, prestação } \\
\text { de contas, monitorização e fiscalização no cumprimento de metas globais de } \\
\text { saúde. A prestação de contas na saúde global tem sido problemática. A WHO e } \\
\text { outras organizações internacionais são criticadas por não estabelecerem metas } \\
\text { detalhadas e realistas. } 0 \text { incremento da transparência em conjunto com os } \\
\text { valores referidos são principios de boa governance. }\end{array}$ \\
\hline
\end{tabular}

Fonte: Elaborado a partir de Gostin e Mok (2009)

Gostin e Mok (2009) constataram que a abordagem da governance global mostrava-se inadequada. As necessidades fundamentais mantinham-se negligenciadas. Como pontos fracos elencavam-se a transparência e a prestação de contas, acompanhadas de monitorização quase inexistente. Perante as fragilidades descritas, os autores referem que a governance global necessita revolver alguns desequilíbrios existentes e tornar a saúde global mais coerente.
Frenk e Moon (2013) referem que, para a saúde global ser fortalecida, é necessário conseguir gerir desafios de governance persistentes. Identificam como desafios a soberania (a saúde continua a ser primordialmente de responsabilidade nacional, no entanto, é cada vez mais difícil que os estados-nações sejam detentores desse controle), o desafio setorial (os processos de formulação de políticas atuais são multissetoriais, no entanto, os atores não estão preparados para assegurar que as necessidades de 
saúde sejam consideradas de forma adequada) e o desafio da prestação de contas.

Apesar de reconhecido que os desafios com que a governance depara não são recentes, é notória a tentativa de fortalecer a prestação de contas no setor da saúde. Têm vindo a ser incluído alguns atores, como organizações da sociedade civil, comunidades de pessoas afetadas por doenças-alvo, e fundações. Essa medida revela uma tentativa de governance mais inclusiva. Para além do descrito, é percebida uma preocupação com o acesso aos medicamentos, e os "defensores" da saúde conseguiram alguma influência na elaboração de políticas para a saúde (Frenk; Moon, 2013).

Ainda que inovadoras, as medidas descritas não são transversais e permanecem limitadas a algumas áreas. Essa limitação é fruto da "luta" que a governance mantém contra os desafios descritos pelos autores. Permanece a necessidade de procurar formas inovadoras de governance global para serem testadas, avaliadas, melhoradas e, se obtiverem sucesso, replicadas. Os desafios que a governance enfrenta continuarão a impedir respostas céleres para a resolução de graves e complexos problemas de saúde em nível global (Frenk; Moon, 2013).

\section{Considerações finais}

As rápidas mudanças políticas, econômicas e sociais desafiaram e continuarão desafiando todas as instituições com papel de liderança a encontrar novas soluções para os problemas do desenvolvimento sustentável.

Em 1997 a UNDP publicou Reconceptualizing governance (UNDP, 1997b), em que já refere a necessidade de encontrar formas mais eficazes para lidar com as questões particularmente importantes de governance - questões que colocaram novos desafios ao Estado na promoção do desenvolvimento humano sustentável, com o reforço do papel da governance na promoção da igualdade de gênero, na redução da pobreza e em uma gestão mais eficiente.

À imagem da governance em geral, a governance na saúde é aplicada em diferentes contextos. Usualmente, o conceito de governance na saúde procura a promoção e a proteção da saúde das populações. Sua aplicação pode ser feita de forma global, com recomendações internacionais, ou de forma local, nomeadamente no nível das organizações. A governance na saúde assume assim um papel de destaque para conseguir responder às necessidades dos complexos sistemas de saúde, desafiados por determinantes da saúde que constantemente os colocam à prova.

Apesar de conhecidos os princípios para a operacionalização da governance, os constantes desafios descritos mantêm a sua incapacidade de resolução dos complexos problemas no setor da saúde. As questões que impedem seu êxito são conhecidas, mas a compreensão universal dos princípios de governance, uma tensão descrita entre a realidade de cada país e as imposições internacionais, a gestão dos desafios persistentes da governance e a ausência de medidas aplicadas de forma transversal continuam a ser fatores que "condenam" o sucesso de sua operacionalização.

Conforme referido, como reação aos desafios impõe-se a necessidade de encontrar formas inovadoras de governance, às quais cabe a difícil tarefa de encontrar respostas numa sociedade que surpreende com desafios sem precedentes. Muitos desses desafios ultrapassam as capacidades e o alcance das instituições tradicionais e de seus processos clássicos de governance. A smart governance (aplicada nos diversos contextos) pode ajudar, tal como referem Scholl e Scholl (2014), a tratar de forma eficaz grandes desafios da sociedade do século XXI e do bem-estar individual. Os autores sugerem que a pesquisa sobre a smart governance deve acompanhar os discursos metafóricos sobre o tema e os projetos práticos existentes, de forma a evitar "caminhos desconhecidos".

Nesse sentido, sugere-se que no futuro a pesquisa procure perceber o impacto desse novo conceito de governance em geral e no setor da saúde em particular.

\section{Referências}

ABDULLAH, H.; VALENTINE, B. Fundamental and ethics theories of corporate governance. Middle Eastern Finance and Economics, Berkeley, v. 4, n. 4, p. 88-96, 2009. 
BARBAZZA, E.; TELLO, J. E. A review of health governance: definitions, dimensions and tools to govern. Health Policy, Amstedam, v. 116, n. 1, p. 1-11, 2014.

BILHIM, J. Qualificação e valorização de competências. Porto: Sociedade Portuguesa de Inovação, 2004.

BILHIM, J. Ciência da administração. Lisboa: Instituto Superior de Ciências Sociais e Políticas, 2013.

BRANDÃO, C. et al. Social responsibility: a new paradigm of hospital governance? Health Care Analysis, New York, v. 21, n. 4, p. 390-402, 2013.

CARRAPATO, P.; CORREIA, P.; GARCIA, B. Determinante da saúde no Brasil: a procura da equidade na saúde. Saúde e Sociedade, São Paulo, v. 26, n. 3, p. 676-689, 2017.

DANILA, R.; MOHAMED, A. M. Assessing governance of the Health Care Delivery System in Malaysia. [S. l.: s. n.], 2013. Disponível em: <https://bit.ly/2IwkPzw>. Acesso em: 10 jun. 2019.

DEIGHAN, M.; BULLIVANT, J. Integrated governance handbook: a handbook for executives and non-executives in healthcare organisations. London: Department of Health, 2006.

EECKLOO, K. et al. From corporate governance to hospital governance: authority, transparency and accountability of Belgian non-profit hospitals' board and management. Health Policy, Amsterdam, v. 68, n. 1, p. 1-15, 2004.

FERREIRA, P. L.; RAPOSO, V. A governação em saúde e a utilização de indicadores de satisfação. Revista Portuguesa de Clínica Geral, Lisboa, v. 22, n. 3, p. 285-296, 2006.

FRENK, J.; MOON, S. Governance challenges in global health. New England Journal of Medicine, Waltham, v. 368, n. 10, p. 936-942, 2013.

GONÇALVES, A. O conceito de governança. In: ENCONTRO DO CONPEDI, 14., 2005, Manaus. Anais... Florianópolis: Conpedi, 2005.

GOODMAN, N. W. Clinical governance. BMJ, London, v. 317, n. 7174, p. 1725-1727, 1998.

GOSTIN, L. O.; MOK, E. A. Grand challenges in global health governance. British Medical Bulletin, Oxford, v. 90, n. 1, p. 7-18, 2009.
JOHNSTON, T. A.; STOUT, S. Investing in health: development effectiveness in the health, nutrition, and population sector. Washington, DC: The World Bank, 1999.

KICKBUSCH, I.; GLEICHER, D. Governance for health in the 21st century. Geneva: World Health Organization, 2012.

KICKBUSCH, I.; GLEICHER, D. Smart governance for health and well-being: the evidence. Copenhagen: WHO Regional Office for Europe, 2014.

KOOIMAN, J. Social-political governance: overview, reflections and design. Public Management: An International Journal of Research and Theory, Abingdon, v. 1, n. 1, p. 67-92, 1999.

NANDA, V. P. The "good governance" concept revisited. The Annals of the American Academy of Political and Social Science, Thousand Oaks, v. 603, n. 1, p. 269-283, 2006.

OPSS - OBSERVATÓRIO PORTUGUÊS DOS SISTEMAS DE SAÚDE. Saúde: que rupturas? Relatório de primavera de 2003. Lisboa: Escola Nacional de Saúde Pública, 2003.

RAPOSO, V. M. D. R. Governação hospitalar: uma proposta conceptual e metodológica para o caso português. 2008. Tese (Doutorado em Organização e Gestão de Empresas) Universidade de Coimbra, Coimbra, 2008.

RHODES, R. A. W. The new governance: governing without government. Political studies, Thousand Oaks, v. 44, n. 4, p. 652-667, 1996.

RODRIGUES, M. Do conceito de governança a uma conceptualização da governança em rede. In: MADUREIRA, C.; ASENSIO, M. (Ed.). Handbook de administração publica. Lisboa: INA, 2013. p. 103-121.

SAKELLARIDES, C. T. El valor de la salud y su "gobierno" en un mundo globalizado posmoderno: el encuentro de la bella y la bestia. Humanitas: Humanidades Médicas, Barcelona, v. 1, n. 3, p. 255-264, 2003.

SCHILLEMANS, T. Moving beyond the clash of interests: on stewardship theory and the relationships between central government departments and public agencies. Public Management Review, Abingdon, v. 15, n. 4, p. 541-562, 2013. 
SCHOLL, H. J.; SCHOLL, M. C. Smart governance: a roadmap for research and practice. In: ICONFERENCE 2014, 2014, Berlin. Anais... Grandville: iSchools, 2014. p. 163-176.

SHORTELL, S. M. New directions in hospital governance. Hospital \& Health Services Administration, Chicago, v. 34, n. 1, p. 7-23, 1989.

SØRENSEN, E. Democratic theory and network governance. Administrative Theory \& Praxis, Abingdon, v. 24, n. 4, p. 693-720, 2002.

STAREY, N. What is clinical governance? Hayward Medical Communications, Kent, v. 1, n. 12, p. 1-8, 2001. Disponível em: <https://bit.ly/2Myu6M2>. Acesso em: 11 jun. 2019.

STOKER, G. Governance as theory: five propositions. International Social Science Journal, Hoboken, v. 5o, n. 155, p. 17-28, 1998.

UNDP - UNITED NATIONS DEVELOPMENT PROGRAMME. Governance for sustainable human development: a UNDP policy document. New York, 1997 .

UNDP - UNITED NATIONS DEVELOPMENT PROGRAMME. Reconceptualizing governance. New York, 1997b. (Discussion paper, 2).

WB - WORLD BANK. World development report 1993: investing in health. New York: Oxford University Press, 1993.

WB - WORLD BANK. Corporate governance: a framework for implementation. Washington, DC, 2000 .
WELSH, D. H. et al. Perceptions of entrepreneurship across generations in family offices: a stewardship theory perspective. Journal of Family Business Strategy, Amsterdam, v. 4, n. 3, p. 213-226, 2013

WHO - WORLD HEALTH ORGANIZATION. Good governance for health. Copenhagen, 1998a.

WHO - WORLD HEALTH ORGANIZATION. Health 21: health for all in the 21st century. Copenhagen, 1998 b.

WHO - WORLD HEALTH ORGANIZATION. Health systems: improving performance. Copenhagen, 2000.

WHO - WORLD HEALTH ORGANIZATION. The European health report 2002. Copenhagen, 2002.

WHO - WORLD HEALTH ORGANIZATION. Working together for health. Copenhagen, 2006. WHO - WORLD HEALTH ORGANIZATION. Health systems financing: the path to universal coverage. Copenhagen, 2010.

WHO - WORLD HEALTH ORGANIZATION. Health 2020: a European policy framework and strategy for the 21st century. Copenhagen, 2013.

WILLKE, H. Smart governance: governing the global knowledge society. Frankfurt: Campus, 2007.

ZARPELON, J. T. G. Governança global na area da saúde e a influência do medo: estudo sobre a OMS e a aids. Relações Internacionais no Mundo Atual, Curitiba, v. 1, n. 15, p. 52-62, 2012.

\section{Contribuição dos autores}

Carrapato concebeu o artigo e o redigiu com Correia, que contribuiu com sua estruturação. Garcia foi responsável pela recolha e análise da bibliografia utilizada.

Recebido: 04/02/2019

Aprovado: 22/05/2019 